\title{
Unusual phase boundary of the magnetic-field-tuned valence transition in $\mathrm{CeOs}_{4} \mathrm{Sb}_{12}$
}

\author{
K. Götze $\odot,{ }^{1, *}$ M. J. Pearce, ${ }^{1}$ P. A. Goddard $\odot,{ }^{1, \dagger}$ M. Jaime, ${ }^{2}$ M. B. Maple, ${ }^{3}$ K. Sasmal,,${ }^{3}$ T. Yanagisawa, ${ }^{4}$ A. McCollam, ${ }^{5}$ \\ T. Khouri, ${ }^{5}$ P.-C. Ho, ${ }^{6}$ and J. Singleton ${ }^{2}$ \\ ${ }^{1}$ Department of Physics, University of Warwick, Coventry CV4 7AL, United Kingdom \\ ${ }^{2}$ National High Magnetic Field Laboratory, Los Alamos National Laboratory, MS-E536, Los Alamos, New Mexico 87545, USA \\ ${ }^{3}$ Department of Physics, University of California, San Diego, La Jolla, California 92093, USA \\ ${ }^{4}$ Department of Physics, Hokkaido University, Sapporo 060-0810, Japan \\ ${ }^{5}$ High Field Magnet Laboratory (HFML-EMFL), Radboud University, Toernooiveld 7, 6525 ED Nijmegen, Netherlands \\ ${ }^{6}$ Department of Physics, California State University, Fresno, California 93740, USA
}

(Received 15 July 2019; accepted 13 December 2019; published 3 February 2020)

\begin{abstract}
The phase diagram of the filled skutterudite $\mathrm{CeOs}_{4} \mathrm{Sb}_{12}$ has been mapped in fields $\mu_{0} H$ of up to $60 \mathrm{~T}$ and temperatures $T$ down to $0.5 \mathrm{~K}$ using resistivity, magnetostriction, and megahertz conductivity. The valence transition separating the semimetallic low- $H$, low- $T \mathcal{L}$ phase from the metallic high- $H$, high- $T \mathcal{H}$ phase exhibits a very unusual, wedge-shaped phase boundary, with a nonmonotonic gradient alternating between positive and negative. The expected "elliptical" behavior of the phase boundary of a valence transition with $H^{2} \propto T^{2}$ originates in the $H$ and $T$ dependence of the free energy of the $f$ multiplet. Here, quantum oscillation measurements suggest that additional energy scales associated with a quantum critical point are responsible for the deviation of the phase boundary of $\mathrm{CeOs}_{4} \mathrm{Sb}_{12}$ from this textbook behavior at high $H$ and low $T$. The distortion of the low- $H$, high- $T$ portion of the phase boundary may be associated with the proximity of $\mathrm{CeOs}_{4} \mathrm{Sb}_{12}$ to a topological semimetal phase induced by uniaxial stress.
\end{abstract}

DOI: 10.1103/PhysRevB.101.075102

\section{INTRODUCTION}

Valence transitions, in which $f$ electrons undergo a temperature- and/or magnetic-field-driven transformation from itinerant to quasilocalized, are associated with significant changes in material properties [1-3] and dramatic alterations to the Fermi surface $[1,4]$. Perhaps the best known is the $\gamma-\alpha$ transition in Ce and its alloys, which leads to a spectacular sample volume collapse [2,3]. Valence transitions are also thought to be responsible for the onset of the "hidden order phase" of $\mathrm{URu}_{2} \mathrm{Si}_{2}$, plus some phase boundaries of elemental $\mathrm{Pu}$ [5-7]. A key identifying feature of valence transitions is the resulting elliptical phase boundary in which the critical magnetic field $H$ and temperature $T$ of the valence transition follow a $H^{2} \propto T^{2}$ behavior (top inset of Fig. 1) or, in other words, lie on a straight line when plotted as $H^{2}$ versus $T^{2}$, with the slope determined by the $g$ factor alone [4].

By contrast, we show here that the valence transition in $\mathrm{CeOs}_{4} \mathrm{Sb}_{12}$, identified by its effect on the Fermi surface and material properties [1], does not follow the above textbook elliptical behavior. We performed megahertz $(\mathrm{MHz})$ conductivity, magnetostriction, and resistivity measurements on $\mathrm{CeOs}_{4} \mathrm{Sb}_{12}$ to map out the phase diagram shown in Fig. 1. The

\footnotetext{
*Corresponding author: k.gotze@warwick.ac.uk

†p.goddard@warwick.ac.uk
}

Published by the American Physical Society under the terms of the Creative Commons Attribution 4.0 International license. Further distribution of this work must maintain attribution to the author(s) and the published article's title, journal citation, and DOI. valence transition separates the low- $T$, low- $H$, semimetallic $\mathcal{L}$ phase and the high- $T$, high- $H$, metallic $\mathcal{H}$ phase [1]; it is immediately obvious that it behaves very unconventionally, falling back to lower $T$ as $H \rightarrow 0$ and lower $H$ as $T \rightarrow 0$. We suggest this unusual behavior is due to the sensitivity of the ground states of $\mathrm{CeOs}_{4} \mathrm{Sb}_{12}$ to quantum fluctuations and proximity to a topological semimetallic phase.

$\mathrm{CeOs}_{4} \mathrm{Sb}_{12}$ is part of an interesting series of rareearth-based filled skutterudites including the unconventional superconductor $\mathrm{PrOs}_{4} \mathrm{Sb}_{12}[12,13]$ and ferromagnetic $\mathrm{NdOs}_{4} \mathrm{Sb}_{12}[1,14]$. While $\mathrm{PrOs}_{4} \mathrm{Sb}_{12}$ and $\mathrm{NdOs}_{4} \mathrm{Sb}_{12}$ possess similar Fermi surfaces comprising multiple pockets (but different effective masses), $\mathrm{CeOs}_{4} \mathrm{Sb}_{12}$ exhibits a valence transition from the heavy-effective-mass (Sommerfeld coefficient $\gamma=92 \mathrm{~mJ} \mathrm{~mol}^{-1} \mathrm{~K}^{-2}$ ) semimetallic $\mathcal{L}$ phase $[9,15-17]$ to the $\mathcal{H}$ phase, characterized by a simple, almost spherical Fermi surface with a light effective mass [1].

Earlier studies [15] suggested $\mathrm{CeOs}_{4} \mathrm{Sb}_{12}$ is a Kondo insulator due to the resistivity increase at low temperatures, but band-structure calculations for the $\mathcal{L}$ phase have confirmed the semimetallic, gapless ground state with heavy masses under ambient conditions and predict the system becomes a topological semimetal or topological Kondo insulator under applied strain [16,17].

Antiferromagnetic order, believed to be due to spin-density wave (SDW) formation, was observed in $\mathrm{CeOs}_{4} \mathrm{Sb}_{12}$ below $1 \mathrm{~K}$ at $H=0[8,18,19]$. The transition temperature $T_{\mathrm{SDW}}$ was seen to increase with increasing field to $2 \mathrm{~K}$ at $7 \mathrm{~T}$ and subsequently to decrease $[1,9,10]$ and be suppressed around $15 \mathrm{~T}$.

In Ref. [1], an attempt to trace the high-temperature limits of the $\mathcal{L}-\mathcal{H}$ boundary was made using $\chi=\partial M / \partial H$ 


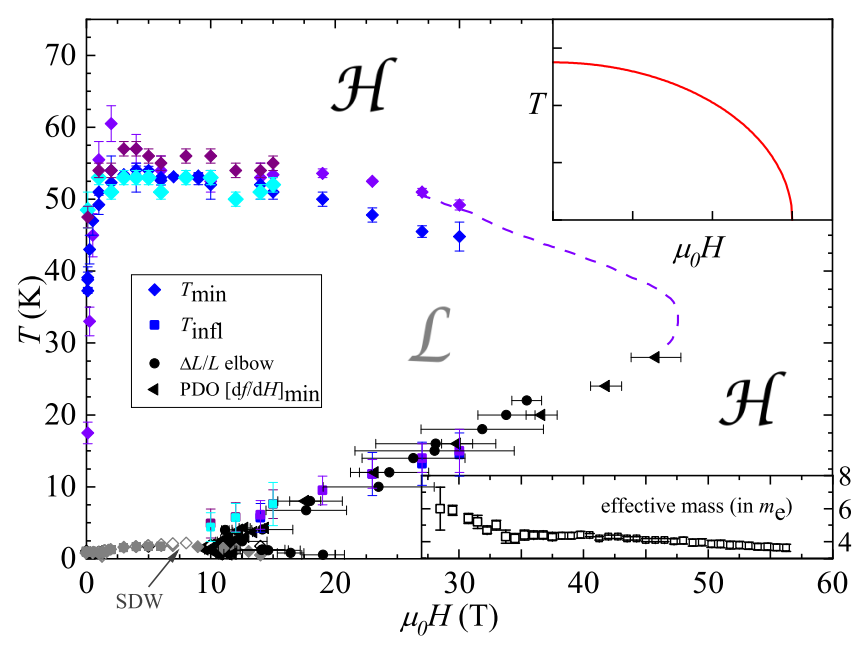

FIG. 1. $T-H$ phase diagram of $\mathrm{CeOs}_{4} \mathrm{Sb}_{12}$ derived from data in this work. Points are from resistivity (solid diamonds: $T_{\min }$, solid squares: $T_{\text {inff }}$; different colors represent different samples), magnetostriction (solid circles), and $\mathrm{MHz}$ conductivity (solid triangles); SDW phase boundary is from Refs. [8-11] (gray symbols). The dotted line is a guide to the eye of a plausible $\mathcal{L}-\mathcal{H}$ phase boundary completion. Top inset: example of the elliptical $H^{2} \propto T^{2}$ phase boundary expected for valence transitions. Bottom inset: field dependence of the effective mass.

contours, where $M$ is the magnetization. However, subsequent experiments suggested that $\chi$ was not an accurate indicator of the valence transition, prompting the current comprehensive series of measurements that reveal the much more unusual behavior shown in Fig. 1.

Our choice of techniques for clarifying the phase boundary is based on previous experimental evidence of the valence transition in cerium-based systems. The strongest indicator of this valence transition is the drastic change in unit cell volume. Magnetostriction measurements indicating the change in sample length as a function of field can very sensitively detect such volume changes and allow for precise determination of the critical field of the phase transition. Owing to the small ratio (1:16) of cerium to other elements in $\mathrm{CeOs}_{4} \mathrm{Sb}_{12}$, we expect a smoother structural transition than the sharp examples observed in elemental or slightly doped cerium [2,3].

Electrical resistivity (and related methods like $\mathrm{MHz}$ conductivity measurements) has been shown to be another reliable indicator of the valence transition in cerium and its alloys: hysteretic behavior in resistivity as a function of temperature or pressure in $\mathrm{Ce}_{0.8} \mathrm{La}_{0.1} \mathrm{Th}_{0.1}$ [3,20] or CeNi [21] occurs at the valence transition due to inhomogeneous strain fields in the sample associated with the cell volume collapse.

\section{EXPERIMENTAL DETAILS}

$\mathrm{CeOs}_{4} \mathrm{Sb}_{12}$ single crystals were prepared using a moltenflux technique [15] with $\mathrm{Sb}$ excess (details can be found in [1]). Several single crystals [cubic space group $T_{\mathrm{h}}^{5}(\operatorname{Im} \overline{3})$, No. 204] were obtained from the same growth batch. Four bar-shaped crystals (samples B1-B4) were used to measure standard four-probe resistivity with the current applied along the [100] axis.
Magnetic fields up to $15 \mathrm{~T}(30 \mathrm{~T})$ were provided by superconducting (water-cooled resistive) magnets. A proximitydetector-oscillator (PDO) technique was used for contactless $(\mathrm{MHz})$ conductivity measurements in pulsed magnetic fields. Shifts in the PDO frequency $f$ are caused by alterations in the sample skin depth [22,23], leading to $\Delta f \propto-\Delta \rho$ for small relative changes in resistivity $\rho$ [22]. Magnetostriction was measured in pulsed fields by the fiber Bragg grating technique [24]. The magnetic field was applied along [001] for all measurements.

\section{RESULTS}

\section{A. Resistivity-temperature sweeps at fixed field}

Figure 2(a) shows $\rho(T)$ for crystals B1-B4 at zero field. In agreement with previous studies $[9,15]$, resistivity initially decreases upon cooling, reaching a minimum at $T=T_{\min }$. Below this temperature, $\rho(T)$ increases strongly. The minimum in $\rho$ was previously interpreted as the $\mathcal{H}-\mathcal{L}$ transition [1]. In contrast to that earlier work, our study shows that the exact value of $T_{\min }$ at $H=0$ is strongly sample dependent, ranging from 17.5 to $48.5 \mathrm{~K}$ for the four crystals measured. Similar sample-dependent variations in the low-temperature magnetic and transport properties of other Ce-based skutterudites were observed in $[25,26]$ and attributed to a delicate balance between competing scattering effects and the influence of magnetic impurities. A more detailed discussion of the sample dependence in our measurements is provided in Sec. IV C.

Note that there is a small but consistent hysteresis between cooling and warming through the $\rho(T)$ minimum, with $T_{\min }$ being higher on cooling than on warming [inset of Fig. 2(a)]. The difference $\Delta T$ increases with temperature-sweep rate, but it is always nonzero even for the slowest temperature changes. With decreasing temperature-sweep rate $\Delta T$ converges to $\approx 0.5 \mathrm{~K}$, indicating that it originates from a phase transition rather than a lag in thermal equilibrium.

As mentioned in the Introduction, similar hysteretic behavior in $\rho$ was observed close to the valence transition in Ce-based materials $[3,20,21]$ but also close to the valence transitions in $\mathrm{YbInCu}_{4}$ [27], supporting the proposal [1] that the valence of $\mathrm{Ce}$ in $\mathrm{CeOs}_{4} \mathrm{Sb}_{12}$ changes at $T_{\min }$.

The shape of the $\rho(T)$ curves changes significantly when a magnetic field is applied. Figure 2(b) shows normalized resistivity curves $\rho(T) / \rho(300 \mathrm{~K})$ for sample B4 for fields up to $15 \mathrm{~T}$. Increasing the magnetic field initially shifts the position of the minimum to higher temperatures; subsequently, $T_{\min }$ is almost field independent between 3 and $15 \mathrm{~T}$ but then decreases [inset of Fig. 2(c)] for higher fields.

The transition to the ordered SDW phase manifests itself by a kink in the $\rho(T)$ curve, marked by down arrows in the inset of Fig. 2(b). Similar features were observed at the transition to the SDW in earlier measurements [9], and the behavior of $T_{\mathrm{SDW}}$ in our data agrees with the results in [9].

For $\mu_{0} H>3 \mathrm{~T}$, a local $\rho(T)$ maximum develops at temperature $T_{\max }$ just above $T_{\mathrm{SDW}}$ [up arrows in the inset of Fig. 2(b)] and moves higher with increasing field. $T_{\max }$ shows an almost linear field dependence (open diamonds in Fig. 3); in addition, as $H$ grows, the $\rho(T)$ maximum becomes broader and lower. These trends continue for fields up to $30 \mathrm{~T}$ [Fig. 2(c)]. 

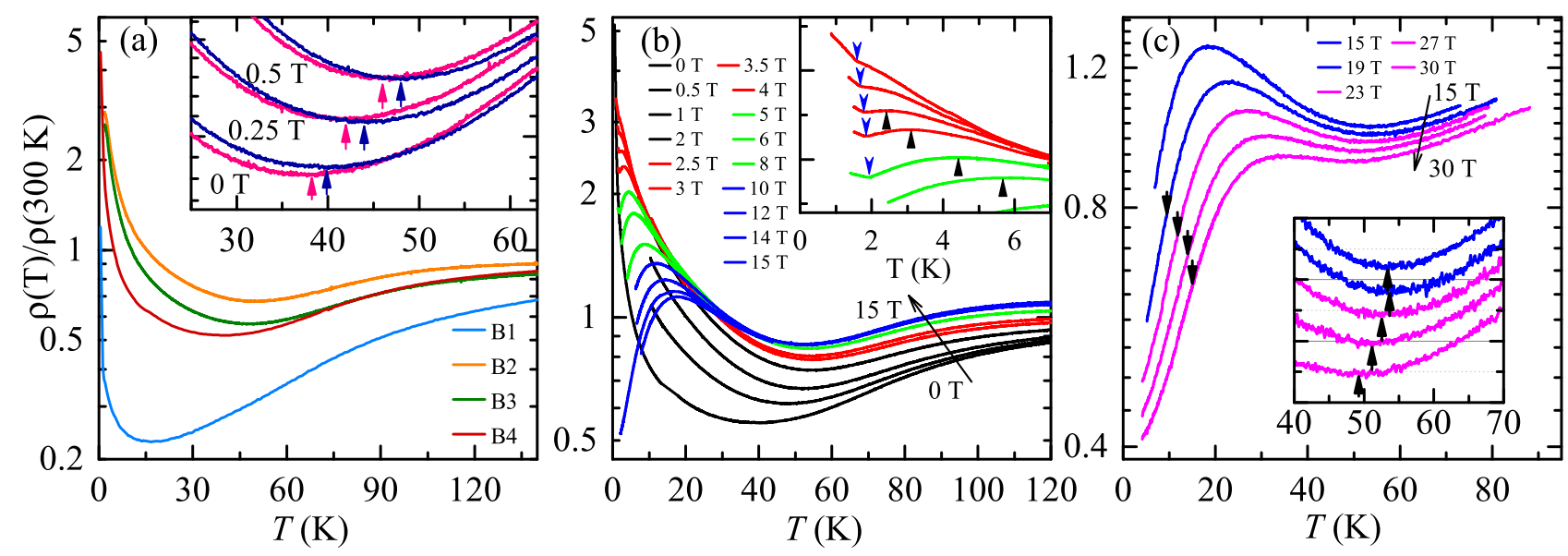

FIG. 2. (a) Resistivity $\rho$ [normalized to $\rho(300 \mathrm{~K})$ ] of all four measured crystals (B1-B4) at $H=0$ for $0.6 \leqslant T \leqslant 140 \mathrm{~K}$. Inset: resistivity hysteresis between warming (red) and cooling (blue) for sample B4 at low fields. Arrows mark the minimum. (b) Normalized $\rho(T)$ of sample $\mathrm{B} 4$ for several fields $\leqslant 15 \mathrm{~T}$. An arrow at high temperatures indicates increasing field; note that the resistivity maximum at low temperatures decreases with increasing field. Inset: low- $T$ behavior of $\rho(T)$ between 2.5 and $6 \mathrm{~T}$. Up arrows mark the emerging maximum for $\mu_{0} H \geqslant 3$ T; down arrows mark the SDW transition. (c) Normalized resistivity of sample B 1 for $15 \leqslant \mu_{0} H \leqslant 30$ T. Curves are offset for clarity. Black arrows mark the inflection point for $\mu_{0} H>15 \mathrm{~T}$. Inset: $\rho$ minimum; arrows track the suppression of $T_{\min }$ with increasing field.

As will be clear from Fig. 1, for fields higher than $10 \mathrm{~T}$, it is possible for a temperature sweep at constant field to traverse the valence transition twice: $\mathcal{H}-\mathcal{L}$, followed by $\mathcal{L}-\mathcal{H}$. In this context, the $\rho(T)$ maximum at $T_{\max }$ is a precursor that occurs before the restoration of metallic behavior at low $T$ and high $H$, but it does not indicate a phase transition. No hysteresis was observed around the maximum, supporting the interpretation that the valence does not change at $T_{\max }$. For $\mu_{0} H \geqslant 10 \mathrm{~T}$, we find a $\rho(T)$ inflection point at $T=T_{\text {infl }}$ below which metallic resistivity $\rho(T)=\rho_{0}+A T^{2}$ is obeyed. It is this inflection point that we attribute to the valence transition. $T_{\text {infl }}$ is marked by down arrows in Fig. 2(c) and indicated by solid squares in Figs. 1 and 3. For fields between $3.5 \mathrm{~T}$, where the $\rho(T)$ maximum first emerges, and approximately $9 \mathrm{~T}$, the SDW phase interposes itself, and $T_{\text {inft }}$ is not visible. Nevertheless, within the region between $T_{\max }$ and

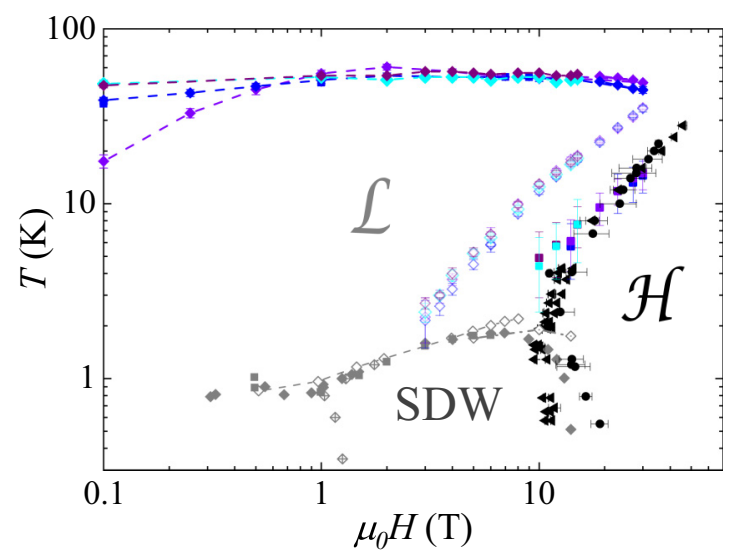

FIG. 3. $T-H$ phase diagram of $\mathrm{CeOs}_{4} \mathrm{Sb}_{12}$ with logarithmically scaled axes. Points are the same as in Fig. 1 with the addition of $T_{\max }$ (open diamonds). $T_{\max }$ does not mark a phase transition, but the precursor of the return to metallic behavior at low $T$ /high $H$.
$T_{\mathrm{SDW}}, \mathrm{CeOs}_{4} \mathrm{Sb}_{12}$ begins to revert to metallic behavior [i.e., $\rho(T)$ falls as $T$ decreases].

\section{B. MHz conductivity and magnetostriction at fixed temperatures in pulsed magnetic fields}

We will now turn to the high- $H$, low- $T$ part of the $\mathcal{L}-\mathcal{H}$ phase boundary that partially coincides with the SDW phase suppression. This portion of the phase boundary was initially identified using PDO experiments for $T \leqslant 4 \mathrm{~K}$ [1]. Here, we use the same technique to track the transition to higher temperatures. Figure 4(a) shows the PDO frequency change in $\mathrm{CeOs}_{4} \mathrm{Sb}_{12}$ in fields of up to $60 \mathrm{~T}$ and temperatures between 0.7 and $40 \mathrm{~K}$. For low temperatures a pronounced maximum exists at low fields, followed by a sharp decrease of $-\Delta f$ and a minimum at high fields. Since $-\Delta f$ is proportional to $\Delta \rho$, we can analyze the PDO $-\Delta f$ data in a manner analogous to how we analyzed the $\rho(T)$ data. The maxima in both properties have the same field dependence, and the PDO maximum continues to move linearly to higher fields with increasing temperature. As in the $\rho(T)$ data, the maximum is the precursor of a change from semimetallic behavior $(\mathcal{L}$ phase) to metallic character ( $\mathcal{H}$ phase) and not a phase transition. The succeeding drop in $\rho$ (or $-\Delta f$ ) is commensurate with removing $\operatorname{Ce} f$ electrons from the $\mathcal{L}$ semimetallic ground state with its ultraheavy effective masses, with the resulting Fermi energy shift producing the larger, almost spherical Fermi surface (with light-mass quasiparticles) of the $\mathcal{H}$ phase predicted by theory and observed in experiment $[1,16,17]$. As in Ref. [1], we identify the $\mathcal{L}-\mathcal{H}$ transition as the inflection point within the fall in $-\Delta f$ [arrows in Fig. 4(a)]. Hysteresis occurs between PDO data recorded with rising and falling field, again suggestive of the lossy kinetics typical of valence transitions [3].

Magnetostriction measurements were carried out in pulsed magnetic fields of up to $60 \mathrm{~T}$ and for temperatures between 0.5 and $40 \mathrm{~K}$ in order to track sample volume changes that 

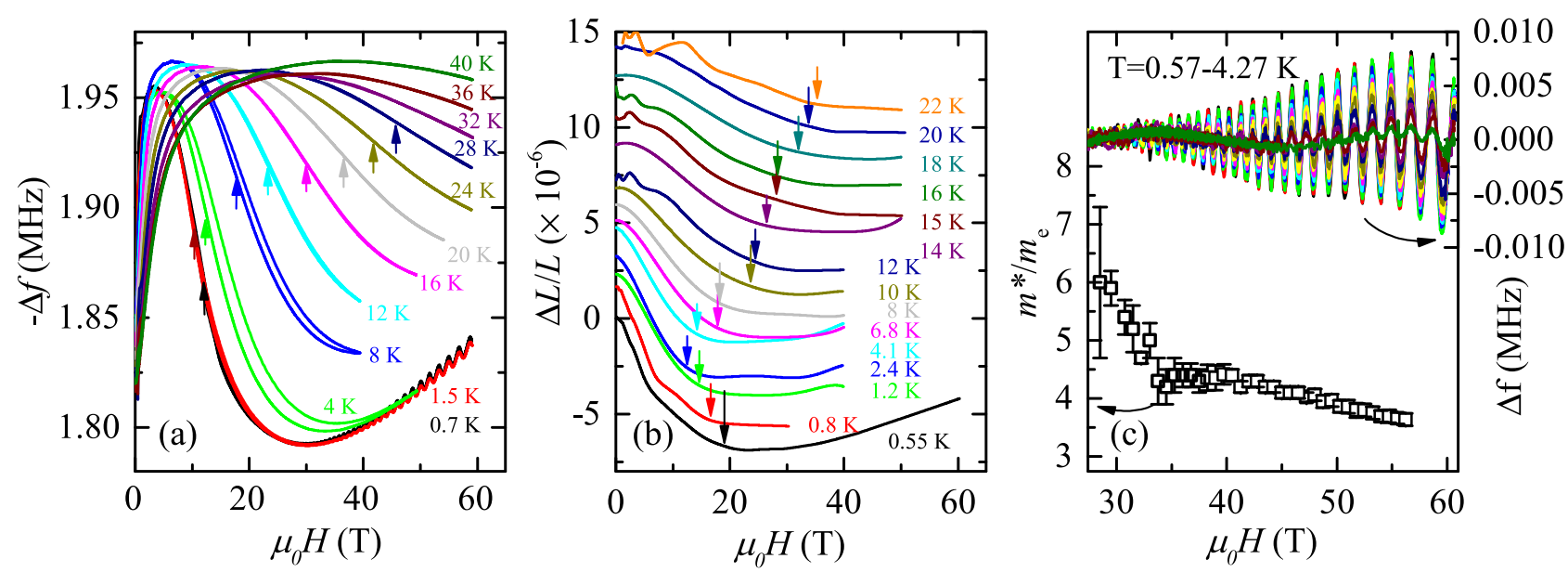

FIG. 4. (a) PDO frequency change $-\Delta f$ in pulsed fields. Note the hysteresis between up and down sweeps. (b) Pulsed-field magnetostriction $\Delta L / L$ (data are offset for clarity). Arrows mark the valence transition in (a) and (b). (c) Oscillatory part of $-\Delta f$ for $0.57 \leqslant T \leqslant 4.27 \mathrm{~K}$, and field dependence of the effective mass of the $F=1.6 \mathrm{kT}$ quantum oscillations; $m_{\mathrm{e}}$ is the bare-electron mass.

accompany the valence transition [2-4]. Typical results are shown in Fig. 4(b) as fractional change in length $(\Delta L / L)$ versus field. The $\mathcal{L}-\mathcal{H}$ phase transition is marked by a change in slope: the initial decrease of $\Delta L / L$ slows down and is reversed or becomes flat, causing an elbow in the data. Note again that only 1 in 17 atoms in $\mathrm{CeOs}_{4} \mathrm{Sb}_{12}$ is cerium, which is expected to lead to a smoother structural transition compared to elemental or slightly doped cerium $[2,3]$.

Linear functions are fitted to the data below and above the elbow; the transition field is defined as the point at which the gradient of the data is equal to the mean gradient of the two linear functions and is indicated by arrows in the graph. Examples of raw and averaged data are shown in Sec. S1 of the Supplemental Material [28]. At low temperatures, the valence transition follows the established SDW border to lower $H$ as $T$ increases [8-11]. Above $4 \mathrm{~K}$ this trend reverses; the transition field starts to grow with $T$. The elbow can be followed up to $22 \mathrm{~K}$; at higher temperatures, it is too weak to be identified reliably.

\section{Quantum oscillations}

Shubnikov-de Haas oscillations in $-\Delta f$ occur in the lower- $T$ curves in Fig. 4(a) above $25 \mathrm{~T}$. These oscillations comprise a single frequency $F \approx 1600 \mathrm{~T}$ due to the roughly spherical $\mathcal{H}$-phase Fermi surface [1]. Using an analysis similar to Ref. [29], the quasiparticle effective mass $m^{*}$ was found to be field dependent. Details and examples can be found in Sec. S2 of the Supplemental Material [28]. Figure 4(c) shows the oscillating part of $-\Delta f$ (right axis) and the development of $m^{*}$ for several mean fields $B_{\mathrm{m}}$ separated by $0.75-\mathrm{T}$ steps. At first, $m^{*}$ increases slowly with decreasing field, from $3.6 m_{\mathrm{e}}$ to $4.4 m_{\mathrm{e}}$ between 56 and $35 \mathrm{~T}$. As the intersection of the $\mathcal{L}$, $\mathcal{H}$, and SDW phases at lower field approaches, $m^{*}$ increases rapidly, reaching $6 m_{\mathrm{e}}$ at $28.5 \mathrm{~T}$, the lowest field at which a value could be determined.

Quantum oscillations in the $\mathcal{L}$ phase have not been observed experimentally. The cross sections of the calculated Fermi surfaces are quite small $(\approx 100$ times smaller than in the $\mathcal{H}$ phase; see Supplemental Material, Sec. S3, for more information [28]), and the quasiparticles are expected to possess heavy masses [15-17]. Very low temperatures would therefore be required to observe quantum oscillations from heavy quasiparticles. However, the presence of the ordered SDW phase below $2 \mathrm{~K}$ would prevent their observation in this temperature regime because of the accompanying Fermi surface reconstruction.

A discussion of whether the field-tuned $\mathcal{L}-\mathcal{H}$ transition might be affected by the quantum limit of quantum oscillations in the $\mathcal{L}$ phase can be found in Sec. S3 of the Supplemental Material [28].

\section{DISCUSSION}

\section{A. Phase diagram}

The $T-H$ phase diagram determined by the PDO, magnetostriction, and transport data $\left(T_{\min }\right.$ and $\left.T_{\text {infl }}\right)$ for B1-B4 is shown in Fig. 1 with linear axes and in Fig. 3 in logarithmic scaling. The SDW phase boundaries were taken from Refs. [8-11]; the high-field data above $10 \mathrm{~T}$ correspond closely to our measurements. The position of the valence transition could be determined quite precisely and agrees for different techniques. Further indications of a thermodynamic phase transition are the hysteresis in transport and PDO and the change in behavior of the lattice indicated by magnetostriction.

It is obvious that the "wedge-shaped" phase boundary surrounding the $\mathcal{L}$ phase is very unusual indeed: on the high$T$, low- $H$ side of the phase diagram, the $\mathcal{H}-\mathcal{L}$ transition temperature $T_{\min }$, as mentioned above, differs for different samples, ranging from 17.5 to $48.5 \mathrm{~K}$ at $H=0$. Additionally, with increasing field for $0 \leqslant \mu_{0} H \leqslant 2 \mathrm{~T}$, the transition at first moves rapidly to higher temperatures (i.e., has a positive gradient). Subsequently, $T_{\min }$ hardly changes between 2 and $15 \mathrm{~T}$ but eventually decreases in higher fields. Among the different samples, the difference between the $T_{\min }$ values decreases above $2 \mathrm{~T}$ but remains at least $4 \mathrm{~K}$ up to $30 \mathrm{~T}$.

On the low- $T$, high- $H$ side, the metallic $\mathcal{H}$ phase is restored. The field-induced $\mathcal{L}-\mathcal{H}$ transition and SDW phase destruction coincide below $T \approx 2 \mathrm{~K}$ and above $10 \mathrm{~T}$. A simple 
interpretation is that the SDW formation is dependent on details of the $\mathcal{L}$-phase Fermi surface topology [30]; once the $\mathcal{L}$ phase is removed by the valence transition, the SDW will inevitably be destroyed. However, we will see below that the death of the SDW and the $\mathcal{L}-\mathcal{H}$ phase boundary are, chicken-and-egg-like, much more subtly entwined than in this simple interpretation. Whereas the low- $T$ part of the high-field phase boundary has a (relatively conventional) negative gradient, above $T=2 \mathrm{~K}$, the phase boundary assumes a positive gradient; the $\mathcal{L}-\mathcal{H}$ transition moves toward higher field with increasing temperature, showing an almost linear $H, T$ relationship up to $28 \mathrm{~K}$.

The local minimum in $\rho(T)$, indicating the $\mathcal{H}-\mathcal{L}$ transition, and the $\rho(T)$ maximum, a precursor to the $\mathcal{L}-\mathcal{H}$ transition, move closer for increasing field, resulting in a plateaulike feature at $30 \mathrm{~T}$ [see Fig. 2(c)]. This convergence shows that the temperature range for which the $\mathcal{L}$ phase is stable shrinks with increasing field. Extrapolating the measured points suggests that above approximately $55 \mathrm{~T}, \mathcal{H}$ should be the only stable phase of $\mathrm{CeOs}_{4} \mathrm{Sb}_{12}$. A logarithmic scaling of the phase diagram displays this behavior more clearly, as shown in Fig. 3 [31].

\section{B. Energy scales and quantum criticality}

The $\mathcal{L}-\mathcal{H}$ phase boundary is clearly different from most others encountered in condensed-matter physics; unlike, e.g., a mean-field boundary [32] or the "domes" observed in many correlated-electron systems such as organic, high- $T_{\mathrm{c}}$, and pnictide superconductors [30,33-35], the gradient of the boundary in $T, H$ space does not change monotonically but alternates positive, negative, positive, negative. By the same token, it clearly deviates from the elliptical $\left(H^{2} \propto T^{2}\right)$ behavior found for field-induced valence transitions in other cerium-based systems [2,3] and uranium compounds [5].

The elliptical phase boundary usually associated with valence transitions is driven by the $T$ and $H$ dependencies of the free energy of the quasilocalized $f$ multiplet [4]. The energies of the Fermi liquids on either side of the phase boundary will depend only slightly on $H$ and $T$, so the multiplet's free energy dominates the situation and drives the valence transition. The $-T S$ (where $S$ is entropy) term in the free energy means the phase in which the multiplet is populated will always be the ground state at high $T$ and high $H[3,4]$, the multiplet's simple partition function resulting in the $H^{2} \propto T^{2}$ elliptical boundary [4]. The deviation of $\mathrm{CeOs}_{4} \mathrm{Sb}_{12}$ from this simple behavior implies that one or more additional energy scales that depend strongly on $H$ and/or $T$ are present.

Turning first to the low- $T$, high- $H$ portion of the valence transition, recall that the $\mathcal{H}$-phase quasiparticle effective mass appears to diverge as the $\mathcal{H}-\mathcal{L}$ transition approaches [Fig. 4(c)]. The antiferromagnetic $\mathrm{PrOs}_{4} \mathrm{As}_{12}$ shows a similar mass increase on approaching the phase boundary of its magnetic ground state [36]. Such effective-mass increases are frequently associated with proximity to a quantum critical point (QCP) $[33,34,36]$. In the case of $\mathrm{CeOs}_{4} \mathrm{Sb}_{12}$, the QCP is most likely associated with the field-driven SDW collapse (Figs. 1 and 3). As $T \rightarrow 0$, the entropy $S$ will diminish as well in accordance with the third law of thermodynamics, leaving any other energy scale to dominate [37]. As a consequence, strong quantum fluctuations-probably antiferromagneticaround the QCP will greatly perturb the Fermi liquids' free energy on either side of the $\mathcal{L}-\mathcal{H}$ boundary [38], challenging the dominance of the multiplet's $-T S$ contribution [4].

\section{Sample dependence}

Moving to the high- $T, H \rightarrow 0$ portion of the $\mathcal{L}-\mathcal{H}$ transition, the most striking feature is the initial, large, positive gradient. Qualitatively similar behavior is seen in the phase diagram of a reduced-dimensionality antiferromagnet [39]; in that case, the effect is attributed to fluctuations affecting the system's free energy. However, the phase-boundary gradient reversal measured in Ref. [39] is much less marked than that in $\mathrm{CeOs}_{4} \mathrm{Sb}_{12}$ (Fig. 1). Moreover, there is no obvious reason why the mechanism of Ref. [39] would yield the strong sample dependence seen here.

The $\mathcal{L}$ phase of $\mathrm{CeOs}_{4} \mathrm{Sb}_{12}$ is thought to be highly unusual among Ce compounds in that a very small application of uniaxial stress can transform it into an unusual topological semimetal [17]. It is therefore possible that the inclusions (fraction of a percent level) of elemental Ce or Os in otherwise very high quality crystals recently observed in neutron scattering experiments [40] result in local regions of varying uniaxial stress inducing topologically protected "domains." The number of domains would likely be very dependent on sample quality, and even small variations in strain within the same batch could give rise to the observed sample dependence. In addition, the band structure associated with such states can be sensitively dependent on magnetic field (see, e.g., Refs. [29,41] and references therein), perhaps leading to the initial positive gradient of the $\mathcal{L}-\mathcal{H}$ boundary. Experimentally, the presence of even a small fraction of topologically protected quasiparticles in an otherwise unremarkable Fermi liquid can have a disproportionate effect on measurable macroscopic properties [29]. Analogous effects may well impact the unusual low- $H$, high- $T$ curvature of the $\mathrm{CeOs}_{4} \mathrm{Sb}_{12}$ valence transition.

\section{SUMMARY}

In summary, the $H-T$ phase diagram of $\mathrm{CeOs}_{4} \mathrm{Sb}_{12}$ has been mapped using resistivity, magnetostriction, and $\mathrm{MHz}$ conductivity. The semimetallic $\mathcal{L}$ phase and the metallic $\mathcal{H}$ phase are separated by a valence transition that exhibits a wedge-shaped phase boundary that is clearly distinct from the textbook elliptical phase boundary usually followed by valence transitions. Field-dependent effective masses revealed by Shubnikov-de Haas oscillations within the $\mathcal{H}$ phase show an increasing $m^{*}$ as the field drops toward the $\mathcal{H}-\mathcal{L}$ phase boundary, suggesting proximity to a QCP. The associated magnetic fluctuations may be responsible for the anomalous $H, T$ dependence of the valence transition at high field. The unusual low- $H$, high- $T$ portion of the phase boundary may, in contrast, be associated with the proximity of $\mathrm{CeOs}_{4} \mathrm{Sb}_{12}$ to a topological semimetal induced by uniaxial stress, resulting in strongly sample dependent behavior.

Data presented in this paper resulting from the UK effort will be made available [45]. 


\section{ACKNOWLEDGMENTS}

We thank R. Johnson for constructive discussions. J.S. would also like to acknowledge useful discussions with Q. Si and P. Coleman. We thank A. Julian and T. Orton for technical assistance. Work performed at the University of Warwick is supported by the European Research Council (ERC) under the European Union's Horizon 2020 research and innovation program (Grant Agreement No. 681260). M.J.P. thanks the EPSRC for additional funding. Work at the National High Magnetic Field Laboratory is supported by NSF Cooperative
Agreements No. DMR-1157490 and No. DMR-1644779, the State of Florida, the US DOE, and the DOE Basic Energy Science Field Work Project Science in $100 \mathrm{~T}$; that at UCSD is supported by NSF Grant No. DMR-1810310 and US DOE Grant No. DEFG02-04ER46105; that at Hokkaido University is supported by JSPS KAKENHI Grants No. JP15KK0146, No. JP18H04297, and No. JP17K05525; that at CSU, Fresno, is supported by NSF Grant No. DMR-1905636, previously NSF Grant No. DMR-1506677. We acknowledge the support of the HFML-RU/FOM, a member of the European Magnetic Field Laboratory (EMFL).
[1] P. C. Ho, J. Singleton, P. A. Goddard, F. F. Balakirev, S. Chikara, T. Yanagisawa, M. B. Maple, D. B. Shrekenhamer, X. Lee, and A. T. Thomas, Phys. Rev. B 94, 205140 (2016).

[2] D. C. Koskenmaki and K. A. Gschneider, in Handbook on the Physics and Chemistry of Rare Earths, edited by K. A. Gschneider and L. Eyring (North Holland, Amsterdam, 1978), Vol. 1, pp. 343-351.

[3] F. Drymiotis, J. Singleton, N. Harrison, J. C. Lashley, A. Bangura, C. H. Mielke, L. Balicas, Z. Fisk, A. Migliori, and J. L. Smith, J. Phys.: Condens. Matter 17, L77 (2005).

[4] M. O. Dzero, L. P. Gor'kov, and A. K. Zvezdin, J. Phys.: Condens. Matter 12, L711 (2000).

[5] N. Harrison and M. Jaime, arXiv:1902.06588.

[6] J. C. Lashley, J. Singleton, A. Migliori, J. B. Betts, R. A. Fisher, J. L. Smith, and R. J. McQueeney, Phys. Rev. Lett. 91, 205901 (2003).

[7] N. Harrison, J. B. Betts, M. R. Wartenbe, F. F. Balakirev, S. Richmond, M. Jaime, and P. H. Tobash, Nat. Commun. 10, 3159 (2019).

[8] T. Namiki, Y. Aoki, H. Sugawara, and H. Sato, Acta Phys. Pol. B 34, 1161 (2003).

[9] H. Sugawara, S. Osaki, M. Kobayashi, T. Namiki, S. R. Saha, Y. Aoki, and H. Sato, Phys. Rev. B 71, 125127 (2005).

[10] C. R. Rotundu and B. Andraka, Phys. Rev. B 73, 144429 (2006).

[11] T. Tayama, W. Ohmachi, M. Wansawa, D. Yutani, T. Sakakibara, H. Sugawara, and H. Sato, J. Phys. Soc. Jpn. 84, 104701 (2015).

[12] E. D. Bauer, N. A. Frederick, P.-C. Ho, V. S. Zapf, and M. B. Maple, Phys. Rev. B 65, 100506(R) (2002)

[13] M. B. Maple, P.-C. Ho, V. S. Zapf, N. A. Frederick, E. D. Bauer, W. M. Yuhasz, F. M. Woodward, and J. W. Lynn, J. Phys. Soc. Jpn. 71, 23 (2002).

[14] P.-C. Ho, W. M. Yuhasz, N. P. Butch, N. A. Frederick, T. A. Sayles, J. R. Jeffries, M. B. Maple, J. B. Betts, A. H. Lacerda, P. Rogl, and G. Giester, Phys. Rev. B 72, 094410 (2005).

[15] E. D. Bauer, A. Slebarski, E. J. Freeman, C. Sirvent, and M. B. Maple, J. Phys.: Condens. Matter 13, 4495 (2001).

[16] H. Harima and K. Takegahara, J. Phys.: Condens. Matter 15, S2081 (2003).

[17] B. Yan, L. Müchler, X.-L. Qi, S.-C. Zhang, and C. Felser, Phys. Rev. B 85, 165125 (2012).

[18] M. Yogi, H. Kotegawa, G.-q. Zheng, Y. Kitaoka, S. Ohsaki, H. Sugawara, and H. Sato, J. Phys. Soc. Jpn. 74, 1950 (2005).
[19] C. Yang, Z. Zhou, H. Wang, J. Hu, K. Iwasa, H. Sugawara, and H. Sato, Rare Met. (Beijing, China) 25, 550 (2006).

[20] J. D. Thompson, Z. Fisk, J. M. Lawrence, J. L. Smith, and R. M. Martin, Phys. Rev. Lett. 50, 1081 (1983).

[21] D. Gignoux and J. Voiron, Phys. Rev. B 32, 4822 (1985).

[22] S. Ghannadzadeh, M. Coak, I. Franke, P. A. Goddard, J. Singleton, and J. L. Manson, Rev. Sci. Instrum. 82, 113902 (2011).

[23] M. M. Altarawneh, C. H. Mielke, and J. S. Brooks, Rev. Sci. Instrum. 80, 066104 (2009).

[24] M. Jaime, C. Corvalán Moya, F. Weickert, V. Zapf, F. F. Balakirev, M. Wartenbe, P. F. S. Rosa, J. B. Betts, G. Rodriguez, S. A. Crooker, and R. Daou, Sensors 17, 2572 (2017).

[25] G. P. Meisner, M. S. Torikachvili, K. N. Yang, M. B. Maple, and R. P. Guertin, J. Appl. Phys. 57, 3073 (1985).

[26] E. D. Bauer, A. Slebarski, R. P. Dickey, E. J. Freeman, C. Sirvent, V. S. Zapf, N. R. Dilley, and M. B. Maple, J. Phys.: Condens. Matter 13, 5183 (2001).

[27] C. D. Immer, J. L. Sarrao, Z. Fisk, A. Lacerda, C. Mielke, and J. D. Thompson, Phys. Rev. B 56, 71 (1997).

[28] See Supplemental Material at http://link.aps.org/supplemental/ 10.1103/PhysRevB.101.075102 for details on the analysis of the magnetostriction data, examples of Lifshitz-Kosevich fits, and a discussion of the absence of quantum oscillations in the $\mathcal{L}$ phase. See also Refs. [15-17,29,42-44].

[29] D. J. Rebar, S. M. Birnbaum, J. Singleton, M. Khan, J. C. Ball, P. W. Adams, J. Y. Chan, D. P. Young, D. A. Browne, and J. F. DiTusa, Phys. Rev. B 99, 094517 (2019).

[30] J. Singleton, Rep. Prog. Phys. 63, 1111 (2000).

[31] The $\mathcal{L}-\mathcal{H}$ transition becomes difficult to observe in pulsedfield PDO data above $28 \mathrm{~K}$, as it merges with the high- $T$ part of the phase boundary close to $40 \mathrm{~K}$. As the upper part of the boundary is very flat in the $T, H$ plot, it is challenging to resolve in any swept-field, constant- $T$ pulsed experiment; the corresponding feature in the data will be very broad. Swept- $T$, fixed- $H$ PDO measurements would be most suitable to follow the flatter, upper boundary to higher fields but would be limited to $\leqslant 30 \mathrm{~T}$ by the resistive magnet used.

[32] P. Chaikin and T. Lubensky, Principles of Condensed Matter Physics, 4th ed. (Cambridge University Press, Cambridge, 2007).

[33] B. J. Ramshaw, S. E. Sebastian, R. D. McDonald, J. Day, B. S. Tan, Z. Zhu, J. B. Betts, R. Liang, D. A. Bonn, W. N. Hardy, and N. Harrison, Science 348, 317 (2015).

[34] B. Michon, C. Girod, S. Badoux, J. Kačmarčík, Q. Ma, M. Dragomir, H. A. Dabkowska, B. D. Gaulin, J.-S. Zhou, S. 
Pyon, T. Takayama, H. Takagi, S. Verret, N. Doiron-Leyraud, C. Marcenat, L. Taillefer, and T. Klein, Nature (London) 567, 218 (2019).

[35] Q. Si, R. Yu, and E. Abrahams, Nat. Rev. Mater. 1, 16017 (2016).

[36] P.-C. Ho, J. Singleton, M. B. Maple, H. Harima, P. A. Goddard, Z. Henkie, and A. Pietraszko, New J. Phys. 9, 269 (2007).

[37] S. J. Blundell and K. M. Blundell, Concepts in Thermal Physics (Oxford University Press, Oxford, 2009).

[38] The strong perturbation of the Fermi-liquid free energy is visibly manifested in the field-dependent effective-mass renormalization.

[39] P. Sengupta, C. D. Batista, R. D. McDonald, S. Cox, J. Singleton, L. Huang, T. P. Papageorgiou, O. Ignatchik, T.
Herrmannsdörfer, J. L. Manson, J. A. Schlueter, K. A. Funk, and J. Wosnitza, Phys. Rev. B 79, 060409(R) (2009).

[40] M. J. Pearce et al. (unpublished).

[41] P. Tang, Q. Zhou, and S.-C. Zhang, Phys. Rev. Lett. 119, 206402 (2017).

[42] J. Singleton, J. W. Kim, C. V. Topping, A. Hansen, E.-D. Mun, S. Chikara, I. Lakis, S. Ghannadzadeh, P. Goddard, X. Luo, Y. S. Oh, S.-W. Cheong, and V. S. Zapf, Phys. Rev. B 94, 224408 (2016).

[43] D. Shoenberg, Magnetic Oscillations in Metals (Cambridge University Press, Cambridge, 1984).

[44] J. Singleton, Band Theory and Electronic Properties of Solid (Oxford University Press, Oxford, 2001).

[45] http://wrap.warwick.ac.uk/131739/. 